RIEF Revue italienne d'études françaises

Littérature, langue, culture

2 | 2012

Varia

\title{
Enfance ou l'impossible mise en mots
}

\section{Giulia Bullentini}

\section{OpenEdition}

Journals

Édition électronique

URL : http://journals.openedition.org/rief/820

DOI : 10.4000/rief.820

ISSN : 2240-7456

\section{Éditeur}

Seminario di filologia francese

\section{Référence électronique}

Giulia Bullentini, «Enfance ou l'impossible mise en mots », Revue italienne d'études françaises [En ligne], 2 | 2012, mis en ligne le 15 décembre 2012, consulté le 19 avril 2019. URL : http:// journals.openedition.org/rief/820; DOI : 10.4000/rief.820

Ce document a été généré automatiquement le 19 avril 2019

\section{(c) (i) $\odot$}

Les contenus de la RIEF sont mis à disposition selon les termes de la Licence Creative Commons Attribution - Pas d'Utilisation Commerciale - Pas de Modification 4.0 International. 


\title{
Enfance ou l'impossible mise en mots
}

\author{
Giulia Bullentini
}

- Alors, tu vas vraiment faire ça ? «Évoquer tes souvenirs d'enfance »... Comme ces mots te gênent, tu ne les aimes pas. Mais reconnais que ce sont les seuls mots qui conviennent. Tu veux « évoquer tes souvenirs »... il n'y a pas à tortiller, c'est bien ça. - Oui, je n'y peux rien, ça me tente, je ne sais pas pourquoi...

1 Enfance a été souvent considérée comme une œuvre atypique dans la production de Nathalie Sarraute, et pourtant elle ne l'est pas. Il s'agit, ici comme ailleurs, de cerner ce qui «palpite faiblement ", des «petits bouts de quelque chose d'encore vivant " $(E$, p. 9). Mais la publication du livre a été accueillie par une prolifération d'articles et de recensions aux tons dépaysés. On se demandait pourquoi la "pionnière » du Nouveau Roman était revenue sur ses pas en abjurant son credo, on ne réussissait pas à s'expliquer comment elle avait pu se laisser couler dans le moule traditionnel de l'autobiographie après avoir salué l'« Ère du Soupçon ». Et pourtant elle n'a pas été la seule, dans le cercle ${ }^{2}$ des Nouveaux Romanciers ${ }^{3}$, à se retourner vers cette forme d'écriture. Butor l'a devancée dans cette démarche: son Portrait de l'artiste en jeune singe. Capriccio (1967) se donne comme le prodrome de la « Nouvelle Autobiographie » néo-romanesque ${ }^{4}$; Robbe-Grillet aussi a emprunté cette direction, et c'est lui qui a théorisé une «Nouvelle Autobiographie $»^{5}$. De toute façon, le choc de la nouveauté - ce qui est parfois inévitable a eu des conséquences néfastes sur la réception de l'œuvre: un défaut (ou mieux un manque) de perspective s'est imposé comme ligne interprétative dominante. En isolant le texte de tout ce qui l'a précédé, on a oublié de faire ressortir les liens qu'il entretient avec la tradition; on a éclipsé son côté classique ou, même, de genre ; on a délaissé sa nature complexe, qui joue avec les stéréotypes et la notion d'authenticité, d'identité, d'appartenance. De cette façon, on a trahi à la fois la dimension toute sarrautienne de l'œuvre et ses dettes avec le modèle du récit autobiographique inauguré par Rousseau ${ }^{6}$. Il est peut-être temps de restituer la parole au dialogue muet des constantes et des variantes qui s'entremêlent sous la surface du texte, de réactiver le paradigme polysémantique que l'auteure a laissé ouvert et que la critique a laissé inexploité. 
2 Intimiste et paradigmatique à la fois, Enfance a la tournure d'une composition musicale à double partition : d'un côté, ces Mémoires réussissent à recréer l'enfance de l'écrivain en restituant le point de vue de l'enfant plongé dans son univers et le pathos de l'adulte qui évoque ses souvenirs avec émotion ; de l'autre côté, ils montrent néanmoins comment les mots excluent tout un monde autre qui a sa propre vie, éphémère mais indispensable à cerner. Comme Kierkegaard ${ }^{8}$, Sarraute ressent le poids et la frustration de toute alternative manquée: elle vise à capturer les mouvements les plus imperceptibles de l'âme avant qu'ils ne se cristallisent en mots, des mots qui expriment mais qui à la fois inévitablement - figent, déforment, dénaturent ${ }^{9}$. C'est là le noyau fondamental et, diraiton, contradictoire de tout l'ensemble de son œuvre : dans la séparation de deux niveaux de la pensée humaine - le niveau du sentiment, des sensations, des émotions, d'une substance indiscernable qui précède la traduction d'une idée en paroles; et le niveau de la mise en mots. On peut, en effet, concevoir l'écriture de Sarraute comme une quête qui vise a récupérer des graines de pensées préexistantes au langage ${ }^{10}$. Il ne faut certainement pas oublier de situer sa recherche à l'intérieur d'une réflexion " moderne » au sens le plus large sur le langage, qui plonge ses racines dans l'Essai sur l'origine des langues (1781) et, notamment, dans l'assertion de Rousseau selon laquelle: "On ne commença pas par raisonner, mais par sentir $»^{11}$. Ce qui est pourtant exceptionnel dans le défi de Sarraute - car elle cherche à trouver une solution en tant qu'écrivain et non pas en tant que philosophe - est le fait de donner vie une dernière fois à la substance palpitante qui serait destinée à mourir par les mots, le fait de lui donner vie précisément par le seul moyen des mots. De cette façon Enfance devient aussi un métadiscours sur ce que l'écrivain ressent comme les limites du langage, des limites qu'elle transcende d'une manière admirable : «cette parole tissée d'approximations et de retouches, de silences et de blancs » (c'est Monique Gosselin qui le remarque) ${ }^{12}$, ces points de suspension, ces tirets sont les armes d'une bataille contre les barrières qui empêchent de dire et de se dire ; ils sont les contours flous, nuancés, tâtonnants, entre le dicible et l'indicible. Phrases suspendues et anaphores ${ }^{13}$ suggèrent, en effet, de nouvelles associations possibles audessous des mots, créant ainsi une sorte d'« occasions » montaliennes, de déchirures dans le filet qui permettent de s'échapper d'un parcours préalablement tracé. Elles visent à dessiner le domaine privilégié des significations possibles, de toutes les potentialités sémantiques que l'actualisation du mot risque d'anéantir. Sarraute - précise encore Gosselin - «ne se contente pas de vouloir dire l'indicible; elle veut faire émerger au seuil du langage, presque sans mots, par une entreprise de transposition sensible, un réel qui affleure mais que tout mot déformerait, écraserait ${ }^{14}$. Un réel constitué par des «mouvements indéfinissables qui glissent très rapidement aux limites de notre conscience ", qui sont « à l'origine de nos gestes, de nos paroles, des sentiments que nous manifestons », qui constituent « la source secrète de notre existence $»^{15}$.

3 Enfance est donc une autobiographie sui generis ${ }^{16}$, qui reste pourtant fidèle au problème essentiel à la base du genre : le souci d'authenticité ${ }^{17}$. Cependant la notion d'authenticité que Sarraute propose est plutôt éloignée de celle que la tradition a fini par fixer comme classique - remarque Deborah Keller : elle est « le contraire d'un réalisme plat ou d'une nostalgie naïve "; elle ne peut pas s'expliquer en dehors d'une stratification de sens qui dérive des significations attribuées dans le temps au même concept par des auteurs tels que Sartre, Nietzsche, Heidegger, Kierkegaard, Camus, Adorno, pour n'en citer que quelques-uns : « ce qui fait la spécificité de l'authenticité sarrautienne, c'est avant tout sa mise à distance du code linguistique. On n'avait rencontré chez aucun des philosophes de 
l'authenticité un tel scepticisme à l'égard de la langue $»^{18}$. Pour comprendre cette spécificité, il faut nécessairement penser aux débuts du genre autobiographique, qui sont inextricablement liés à l'invention de la « gratuité ». Dans un livre fondamental pour les études sur le récit d'enfance, Infanzia, Memoria e Storia, Francesco Orlando saisit toute la portée de la révolution rousseauienne en la synthétisant dans une série de binômes antinomiques, dont le principal est : souvenirs d'enfance gratuits vs souvenirs motivés par la narration $^{19}$. Dans Les Confessions, on trouve évidemment de nombreux exemples des deux cas : lorsque Jean-Jacques raconte l'épisode de la fessée de Mlle Lambercier, c'est pour y découvrir l'imprinting de sa vie sexuelle adulte. Par contre, lorsqu'il parle au lecteur de la chanson que sa tante Suson lui chantait quand il était enfant, et qu'il la transcrit sur la page en gardant les lacunes dessinées par l'oubli, et qu'en même temps il s'émeut et se berce dans le plaisir du souvenir, dans ce cas-là, il nous offre un souvenir d'enfance gratuit: sans rapport explicite avec le développement de sa personnalitéro. S'il s'abandonne à l'émotion du souvenir, c'est gratuitement, ce n'est que pour avoir la sensation - étant vieux déjà - de ressaisir la vie par ses origines, de recommencer depuis le début. Avant Les Confessions, tout cela n'aurait même pas été pensable : et ce n'est pas par hasard que ces Mémoires ont été enfantés au moment où la conception chrétienne traditionnelle de la mort commençait à s'affaiblir, le transcendant laissait sa place à l'immanent. Orlando l'explique bien: «Le pathos qui investit à partir de Rousseau l'extrémité initiale de la vie humaine provenait de la partie finale de l'existence; il était emprunté au vieillissement et à la mort, calqué sur l'attente de l'au-delà ${ }^{21}$. René Girard, lui aussi, a condensé tout cela en une brillante formule: "À mesure que le ciel se dépeuple, le sacré reflue sur la terre $\aleph^{22}$. Or, Sarraute vit sous un ciel complètement dépeuplé, dans un univers post-révolutionnaire et post-freudien : elle a tout à fait le droit de raconter son enfance, même dans les détails les plus insignifiants, et de s'émouvoir pendant qu'elle le fait; mais elle ne pourra plus jamais prétendre à la gratuité absolue. Rien n'est gratuit depuis que Freud a expliqué que «dans les trois ou quatre premières années de la vie se fixent des impressions et s'établissent des modes de réaction au monde extérieur, qu'aucun événement ultérieur ne peut plus dépouiller de leur force $»^{23}$. Sur un plan ontogénétique, même l'événement le plus banal et le plus fortuit de notre enfance contribue à fixer notre destinée, c'est-à-dire à structurer notre caractère selon une façon particulière de désirer, et il donne ainsi un sens à nos vies. Ce qui vient après n'est qu'une compulsion de répétition. Sur un plan phylogénétique, la matière qui constitue l'enfant ce qui est futile, ordinaire, inconséquent - devient fonctionnelle, car il ne s'agit plus d'un souvenir ému qui ne serait cher que pour celui qui le raconte, mais d'un instrument pour l'autoscopie d'une civilisation entière: chaque détail, bien qu'il soit insignifiant en apparence (mais seulement en apparence, car les rapports de causalité demeurent cachés), devient potentiellement dépositaire, en effet, d'un savoir refoulé, à l'intérieur de ce que Carlo Ginzburg définit comme le "paradigme indiciaire "24 moderne. En conséquence, bien que Sarraute raconte encore des épisodes qui n'ont aucune fonction à l'intérieur de la logique du récit (tout comme l'aurait fait Rousseau), son lecteur désormais rusé - ne peut plus croire ingénument à l'existence d'un domaine du gratuit. Car, s'il existe un long parcours à travers lequel la littérature occidentale est arrivée à représenter l'individu en tant que fonction de l'histoire ${ }^{25}$, il en existe un autre aussi, parallèle au premier, à travers lequel l'autobiographie est arrivée à représenter ce même individu en tant que fonction de son enfance. Ce n'est là, d'ailleurs, qu'un corollaire de cette relation entre ontogenèse et phylogenèse dont nous parlions ci-dessus. Ce parcours, 
nous l'avons dit, c'est Rousseau qui l'a entrepris le premier; et le texte de Sarraute en est une étape fondamentale, comme le montrent ces quelques lignes:

« Nein das tust du nicht »... «Non, tu ne feras pas ça »... les voici de nouveau, ces paroles, elles se sont ranimées, aussi vivantes, aussi actives qu'à ce moment, il y a si longtemps, où elles ont pénétré en moi [...] je ne peux pas me voir, mais je le sens comme si je le faisais maintenant... je saisis brusquement les ciseaux, je les tiens serrés dans ma main... des lourds ciseaux fermés... je les tends la pointe en l'air vers le dossier d'un canapé recouvert d'une délicieuse soie à ramages, d'un bleu un peu fané, aux reflets satinés... et je dis en allemand... « Ich werde es zerreissen. » [...] « Je vais le déchirer »... [...] «Non, tu ne feras pas ça...» les paroles m'entourent, m'enserrent, me ligotent, je me débats... «Si, je le ferai ». (E, p. 10-13)

Voilà le premier souvenir d'enfance que Sarraute offre à son lecteur - sans aucune raison, en apparence : ce n'est pas le plus ancien et il n'entre pas dans un rapport de cause à effet avec ce qu'on lira par la suite. Et pourtant comme on est loin de la gratuité du type rousseauien! Si on lit Enfance en tant qu'autobiographie, cet épisode remplace une naissance dont on ne saurait se souvenir. Il ne trahit d'ailleurs pas la nature traumatique d'un tel événement : la déchirure par les ciseaux symbolise très bien la violence originelle du moment où - comme le dirait Chateaubriand - notre mère nous « infligea la vie $»^{26}$, en nous condamnant à un exil continuel et à un malheur éternel. Par conséquent, il a une valeur initiatique déterminante, qui s'affermit encore plus si on pense que les mots sont au cœur de cette évocation, qu'ils sont le seul objet d'intérêt véritable pour l'écrivain adulte. La gratuité disparaît pour faire place à la surdétermination : l'enfance se donne à Sarraute comme un point de vue privilégié pour saisir le moment où les abus de la société débutent, les abus de la langue sur l'ensemble informe des pensées qui cherchent à se constituer en identité individuelle. L'enfance révèle le moment où tout se joue dans un domaine à la limite « entre l'impression et la sensation, dans ce flou que les mots essaient de cerner, d'apprivoiser $\aleph^{27}$, là où s'accomplit le miracle à travers lequel le sujet se forme par le dialogue avec soi-même et les autres. Ce n'est peut-être pas par hasard qu'on trouve ici le terme zerreissen, dont la racine germanique a donné également le mot anglais to write: écrire. Car, en soixante-dix scènes ${ }^{28}$ qui se disposent sur la page comme un poème en prose, Sarraute nous rend spectateurs de la naissance d'une voix s'affirmant contre les abus et contre le pouvoir aliénant du langage. Cette voix, sa voix, c'est l'écriture. Enfance est donc un récit tout spécial, un récit où du moi qui se raconte ne reste qu'une voix. Une voix qui interagit avec une autre voix, qui «se schize $»^{29}$, qui extrait avec des capacités maïeutiques - les souvenirs et les réflexions du brouillard de l'oubli, selon " une configuration méta-analytique féconde de ses ambiguïtés $»^{30}$. Ou, pourrait-on dire aussi, un moi dédoublé qui se parle et se répond solipsistiquement, en transformant le monologue en dialogue :

- [...] Mais maintenant quand je m'efforce de reconstituer comme je peux ces instants, ce qui me surprend d'abord, c'est que je ne retrouve pour ainsi dire pas de colère ou de rancune contre «l'oncle ».

- Il y a dû en avoir pourtant... Il avait été brutal...

- C'est sûr. Mais elle s'est probablement très vite effacée et ce que je parviens à retrouver, c'est surtout une impression de délivrance... un peu comme ce qu'on éprouve après avoir subi une opération, une cautérisation, une ablation douloureuses, mais nécessaires, mais bienfaisantes...

- Il n'est pas possible que tu l'aies perçu ainsi sur le moment...

- Évidemment. Cela ne pouvait pas m'apparaître tel que je le vois à présent, quand je m'oblige à cet effort... dont je n'étais pas capable... quand j'essaie de m'enfoncer, d'atteindre, d'accrocher, de dégager ce qui est resté là, enfoui. (E, p. 85-86) 
C'est là, précisément, que réside la grande nouveauté de cette autobiographie : dans cette modification profonde de la façon de se raconter. Ce que Philippe Lejeune définissait comme un « récit rétrospectif en prose qu'une personne réelle fait de sa propre existence, lorsqu'elle met l'accent sur sa vie individuelle, notamment sur l'histoire de sa personnalité $\aleph^{31}$ a évolué dans une sorte de combat, d'affrontement à deux. Bien que la critique ait largement souligné l'importance de cet aspect (il suffit de penser aux études de Doumet et Valenta) ${ }^{32}$, on n'a peut-être pas encore assez réfléchi sur la multiplicité extraordinaire de significations qui se cachent derrière cet expédient diégétique. En premier lieu, l'effet de résonance stéréoscopique créé par le moi et son double se donne comme un tribut offert à la tradition. Paradoxalement, ce qui a l'allure d'une extrême modernité - cette simulation d'une séance psychanalytique, ce jeu dans lequel une poignée de tirets se chargent de démêler le va-et-vient schizophrénique entre les différentes parties d'une même conscience, ce voyage hypnotique à la reconquête d'un passé insaisissable - c'est en fait une marque de fidélité aux problématiques incontournables du genre, notamment, au topos de la mémoire infidèle et de l'oubli insidieux. Orlando synthétise ce souci originaire de tout autobiographe dans l'opposition entre deux autres termes, deux voies alternatives par lesquelles le moi s'approche de la matière du passé et de ses pièges: abandon au souvenir vs critique du souvenir ${ }^{33}$. Ce binôme antinomique est indispensable pour saisir, à la fois, la distance et le lien secret de Sarraute par rapport à ses prédécesseurs. Afin de comprendre en profondeur l'importance de cette bipartition, il faut se référer encore une fois à Rousseau, qui a " inventé » le paradigme du récit d'enfance et semble en avoir prévu, en quelque sorte, les développements à venir. On remarquera que dans Les Confessions il n'y a qu'un seul terme (l'autre restant virtuel) de l'opposition : à savoir l'abandon pathétique. La grande nouveauté de Rousseau a consisté précisément à rendre l'hommage de l'émotion au souvenir d'enfance. Stendhal, par contre, a accordé une grande place à l'instance critique, en soumettant chacun de ses souvenirs à une enquête philologique sans fin. Chez lui, on voit bien le deuxième terme de la distinction orlandienne : la Vie de Henry Brulard est entièrement parsemée de plans, dessins, lettres capitales qui indiquent des positions, des distances, des directions. On voit à chaque pas un effort authentique pour rejoindre la vérité objective. Ou, comme le dit Orlando : pour «arriver à des datations, traverser des stratifications, remédier aux lacunes, rectifier les jugements enfantins, en intégrer la perspective limitée $»^{34}$. Enfance porte les traces de cette même quête incessante de la vérité jusque dans sa structure. Le jeu stéréophonique des deux voix qui s'interrogent, se répondent, en transformant le récit dans une sorte de dramaturgie, ce jeu est l'emblème diégétique de la critique philologique du souvenir :

«Ce n'est pas ta maison »... On a peine à le croire, et pourtant c'est ce qu'un jour Véra m'a dit. Quand je lui ai demandé si nous allions bientôt rentrer à la maison, elle m'a dit : «Ce n'est pas ta maison. »

- Tout à fait ce que la méchante marâtre aurait pu répondre à la pauvre Cendrillon. C'est ce qui t'a fait hésiter...

- En effet, je craignais qu'en revivant cela, je ne me laisse pousser à faire de Véra et de moi des personnages de contes de fées...

- Il faut dire que Véra, par moments, quand on s'efforce de l'évoquer, donne le sentiment de décoller du réel, de s'envoler dans la fiction...

Mais ne pourrait-on pas, cette fois, pour se maintenir dans la réalité, essayer d'imaginer que ces paroles, elle les a prononcées parce qu'il restait entendu que ma mère allait me reprendre, il ne fallait pas trop m'habituer à me sentir chez moi dans 
une maison que je devrais bientôt quitter... elle voulait m'éviter un nouveau déchirement... (E, p. 130-131)

6 En introduisant l'idée si originale d'un double à moitié entre un autre soi-même et un autre par rapport à soi-même, l'écrivain s'est admirablement approprié un topos de matrice stendhalienne tout en le réinventant. Elle l'a réinventé en cherchant dans le souvenir, non pas les vestiges matériels de quelque chose qui a eu lieu, mais bien plus les traces immatérielles des pensées qui ont existé avant d'être traduites en mots, hors des mots $^{35}$. D'où, l'impression d'une scène qui, loin d'être réelle, a plutôt des contours nuancés; une scène qui fait émerger, par petits coups de pinceau, doucement, le bon mot : celui qui ne tuerait pas la pensée informe du subconscient ${ }^{36}$, qui échapperait à ce que l'écrivain conçoit comme la menace d'hypostatisation représentée par le langage - car «toute mise en mots risque de devenir une mise à mort $»^{37}$. Ces quelques lignes sont emblématiques :

Ce qui passait entre Kolia et maman, ce courant chaud, ce rayonnement, j'en recevais, moi aussi, comme des ondes...

- Une fois pourtant... tu te rappelles...

- Mais c'est ce que j'ai senti longtemps après... tu sais bien que sur le moment...

- Oh, même sur le moment... et la preuve en est que ces mots sont restés en toi pour toujours [...].

- Maman et Kolia faisaient semblant de lutter, [...] et j'ai voulu participer, [...] j'ai passé mes bras autour d'elle comme pour la défendre et elle m'a repoussée doucement... «Laisse donc... femme et mari sont un même parti.» Et je me suis écartée...

- Aussi vite que si elle t'avait repoussée violemment.

- Et pourtant sur le moment ce que j'ai ressenti était très léger... c'était comme le tintement d'un verre doucement cogné...

- Crois-tu vraiment?

- Il m'a semblé sur le moment que maman avait pensé que je voulais pour de bon la défendre, que je la croyais menacée [...].

Et c'est tout? Tu n'as rien senti d'autre? Mais regarde... maman et Kolia discutent, s'animent, ils font semblant de se battre, ils rient et tu t'approches [...] Allons, fais un effort... (E, p. 73-75)

7 L'ensemble des répliques a, peut-on dire, la fonction de glisser l'ombre du doute dans l'édifice tout lisse et inattaquable de l'enfance racontée. Si Stendhal avait déjà ressenti la nécessité de soumettre les données de sa mémoire au tri d'une conscience critique rigoureuse, s'il l'avait fait en fouillant les archives de famille, en esquissant les lignes de l'espace et $\mathrm{du}$ temps avec une précision mathématique, au $\mathrm{XX}^{\mathrm{e}}$ siècle, ce souci d'exactitude se charge d'autres significations: la nécessité de ne pas figer le flou qui précède la conscience du moi, l'intention de montrer la pensée en formation, la volonté de prévenir la cristallisation que les mots opèrent sur les frêles illuminations de l'esprit. Enfance - on a insisté sur ce point - se compose autour d'un difficile équilibre entre tradition et innovation, autour d'un dialogue qui procède d'écart en écart par rapport à des règles codifiées dans le temps et ratifiées par les habitudes de lecture. Philippe Lejeune - qui a consacré maintes de ses études à explorer les contours de l'autobiographie 38 - est l'un des rares critiques qui l'ont remarqué : "Enfance constitue un retour à la tradition, c'est un livre très classique. Mais Nathalie Sarraute fait retour avec armes et bagages. Rien d'une apostasie, ni d'une chute. Plutôt une opération de sauvetage : elle sauve le genre du récit d'enfance... des atteintes de la veillesse ${ }^{39}$. Ou encore : "Enfance est un texte engendré par les textes précédents, autant qu'un texte qui révèle ce qui les engendra $»^{40}$. 
Cette forme de non-apostasie constitue donc le deuxième fondement de la structure dialogique: Sarraute poursuit, même dans son autobiographie, son enquête sur les tropismes. D'ailleurs, le dédoublement du moi qui se parle et se répond avait été préconisé en quelque sorte dans Entre la vie et la mort, où un spectateur pluriel externe au sujet assistait à cette scission perturbante :

On dirait qu'il se scinde, se dédouble. Une moitié de lui-même, déléguée auprès de nous, prend place parmi nous dans le cercle, avec nous à distance contemple, interroge... ensemble nous cherchons à percer le mystère, à expliquer le miracle. L'autre moitié restée au milieu du cercle s'efforce comme elle peut de nous aider... ${ }^{41}$

Dans ses romans, Sarraute avait voulu mettre en scène des instances décharnées, les observer dans leurs affrontements, combiner l'aliénation inévitable du langage avec les rapports de force interpersonnels. En publiant Enfance, elle reste parfaitement cohérente et fidèle à sa production précédente, elle en atteint même le comble: le microscope se tourne sur l'écrivain, mais il n'a pas perdu son pouvoir révélateur. L'interaction de deux voix empêche le moi de glisser dans l'énonciation des significations postiches où il n'y avait qu'un mouvement, presque imperceptible :

[...] mais cette reconstitution de ce que j'ai dû éprouver est pareille à une maquette en carton reproduisant en un modèle réduit ce qu'avaient pu être les bâtiments, les maisons, les temples, les rues, les places et les jardins d'une ville engloutie...

- Pas entièrement...

- Quelque chose s'élève encore, toujours aussi réel, une masse immense... l'impossibilité de me dégager de ce qui me tient si fort, je m'y suis encastrée, cela me redresse, me soutient, me durcit, me fait prendre forme [...]

- Il est curieux que tu aies éprouvé précisément le sentiment que l'enseignement primaire cherchait à donner...

- J'ai été étonnée en apprenant beaucoup plus tard que c'était là un des buts que cet enseignement voulait atteindre. En tout cas, avec moi, il y a réussi.

- L'école dominait ton existence... elle lui donnait un sens, son vrai sens, son importance... Quand tu t'es sentie si malade, tu avais la rougeole, tu as prié le Ciel... (E, p. 173-174)

Cette espèce de stichomythie ne vise pas à ressaisir des bribes de passé qui se sont échappées par les mailles du temps, mais plutôt à éviter le péril d'une hypostatisation de la conscience, ce qui est encore plus faux dans le cas de l'enfance. Il est surprenant de noter la ressemblance extraordinaire du passage qui décrit la maquette en carton de la mémoire avec la célèbre page proustienne de la madeleine, où, comme en un jeu japonais, villes et jardins et visages sortent d'une tasse de thé. Une fois encore il faut noter la présence insistante des marques de code et de genre à l'intérieur d'un discours aussi original, personnel et nouveau. Le rapprochement avec Proust permet de replacer le texte dans une dialectique féconde avec l'histoire qui l'a précédé. Et cela notamment par rapport au statut du regard porté sur les souvenirs : si classique et pourtant si moderne, chez Sarraute.

11 Parmi les couples de constantes qui qualifient le thème des souvenirs d'enfance, Orlando en a repéré un autre qui oppose les différentes perspectives que le mémorialiste adulte projette sur le paysage de sa mémoire : regard en arrière vs regard d'en bas. Les deux expressions, on le sait bien, viennent de Stendhal ${ }^{42}$. Le regard en arrière, que l'autobiographe plus ou moins âgé tourne vers son enfance avec tendresse, est - si je peux m'exprimer d'une façon un peu simpliste - la modalité la plus automatique et la plus fréquente de recréer son passé : il est souvent introduit par le biais de ce «je revois encore » qui est devenu, par la suite, l'emblème du récit rousseauien. Le regard d'en bas, 
par contre, se situe idéalement du côté de la modernité : il donne consistance aux tentatives de restituer la perception que l'enfant, à cause de son âge et de sa taille, avait connue des réalités physiques et morales plus grandes que lui. Il est le fruit d'une expérimentation stylistique complexe : il faut que les mots s'adaptent aux proportions du moi-enfant, qui juge et comprend moins ou, pour mieux dire, d'une façon autre par rapport aux adultes. Il s'agit, à chaque fois, de filtrer la narration à travers une ironie spéciale ou de porter sur les objets un regard "dépaysé ", en leur donnant des désignations nouvelles, vierges. On sait que les exemples de ce procédé stylistique ne manquent ni chez Rousseau ni chez Stendhal, mais c'est au XXe siècle qu'il y a eu un tournant significatif dans ce sens : des écrivains comme Joyce (dans Dedalus) et Sartre (dans Enfance d'un chef) arrivent à se couler totalement dans le point de vue de l'enfant, d'après une perspective qui est spatiale et mentale à la fois. Cette tradition - il faut le répéter - Sarraute a su la métaboliser pour créer quelque chose d'absolument nouveau. Le lecteur d'Enfance est le témoin d'un effort constant et authentique pour voir les choses "d'en bas", ce qui se réalise surtout dans une reconquête du rapport que l'enfant instaure avec les mots : il y a les «mots de chez moi, des mots solides que je connais bien » $(E$, p. 87$)$; et il y a les mots des autres, toujours meurtriers, des mots qui cherchent à se glisser dans la conscience pour l'aliéner à elle-même, pour anéantir la volonté du moi. On a dit que le choix même de donner à la narration une structure par scènes, au lieu de la coudre dans un récit unitaire, est significatif: il permet aux tropismes de revivre dans leur forme originale, et il empêche les interprétations postiches que l'écrivain adulte pourrait être tenté d'en donner. Le risque est réel, parce que les objets du souvenir sont étrangers et insaisissables pour les processus de la mémoire ${ }^{43}$ - qui peuvent se présenter comme soudains et involontaires, ou bien comme complexes et lents. Mais Sarraute ne se laisse pas prendre au piège : elle décrit à la fois le fonctionnement de la mémoire volontaire (l'intention d'« évoquer des souvenirs»), celui de la mémoire involontaire (l'effort de trouver une signification à la tentation), et le lien qui rattache celle-ci à l'objet du souvenir (le besoin de faire revivre les tropismes de l'enfance avant qu'ils disparaissent ${ }^{44}$ ). Entre la mémoire volontaire et la mémoire involontaire, elle fait glisser les lents mouvements du subconscient qui hantent toujours ses pages - les courants tropismaux ${ }^{45}$. À travers ce biais, les processus se ressoudent aux objets de la mémoire. Car les tropismes gardent les traces des empreintes enfoncées par les petits faits du dehors, ils naissent dans l'enfance, ils sont l'enfance : c'est-à-dire, au sens étymologique, ce qu'on ne peut dire. Comme le remarque très pertinemment Keller : «L'expression artistique passe par une tentative de retrouver le regard premier de l'enfant ainsi que par une mise en cause systématique, par des procédés d'ironie, de tout ce qui relève du stéréotype $»^{46}$. Chez Sarraute, le langage vise à défier l'ineffable; et il réussit dans son but en s'élevant - ou plutôt en s'abaissant - à la perspective enfantine selon un mélange de tonalités tout spécial. En essayant de démêler les différents fils de cette combinaison, on y découvrira une première composante de provenance décidément rousseauienne, qui fusionne ironie et émotion. Il s'agit presque d'un clin d'œil au lecteur qui connaît les modèles littéraires auxquels l'auteur fait référence: Loti, Boylesve ${ }^{47}$, les Confessions. Si on se souvient de l'épisode du noyer, du ton héroï-comique par lequel Jean-Jacques chante les mésaventures de deux enfants qui plantent un arbre et l'arrosent à l'insu des adultes, quitte a être dévoilés ensuite; si on se souvient de l'ironie qui tamise l'émotion de l'adulte qui raconte, on reconnaîtra aisément tout cela dans les lignes suivantes d'Enfance, où le noyer est devenu une pastèque : 
La tête couverte d'un long voile de mousseline blanche [...], je conduis la procession qui porte en terre une grosse graine noire et plate de pastèque. Elle repose dans une petite boîte sur une couche de mousse... nous l'enterrons [...], nous l'arrosons avec notre petit arrosoir d'enfant, j'agite au-dessus de la terre ma baguette magique en prononçant des incantations faites de syllabes barbares et drôles que j'ai longtemps retenues et que je n'arrive plus à retrouver... Nous irons nous pencher sur cette tombe jusqu'au jour où enfin nous aurons peut-être la chance de voir sortir de terre une tendre pousse vivante... $(E$, p. 33-34)

Cependant il y a aussi une empreinte plus typiquement sarrautienne, qui consiste à faire émerger des profondeurs de l'oubli le langage du souvenir. Pour cela il suffira de repenser à l'épisode du canapé déchiré ( $E, \mathrm{p} .10-13)$. Par l'évocation de cette scène, le lecteur revit avec l'enfant tout le drame de la violence des mots, ces mots précis, dans une langue que Natacha (son nom en russe) n'a plus jamais entendue après cela. Et la présence de ces phrases en allemand, dans un souvenir tellement significatif qu'il forme l'incipit du livre, ne saurait être fortuite ou gratuite : l'écrivain en fait des instruments pour démontrer sa thèse, pour révéler que les mots font toujours violence au sens. Et cela inévitablement, car ils sont toujours étrangers à ce qui a une substance autre, à ce qui est pré-linguistique au sens rousseauien, pourrait-on dire. Le langage du souvenir d'enfance coïncide évidemment avec la voix de l'enfant, comme le montrent très bien les lignes suivantes :

Nous nous promenons je ne sais où à la campagne, maman avance doucement au bras de Kolia... je reste en arrière plantée devant le poteau de bois... «Si tu le touches, tu meurs ", maman a dit ça... J'ai envie de le toucher, je veux savoir, j'ai très peur, je veux voir comment ce sera, j'étends ma main, je touche avec mon doigt le bois du poteau électrique... et aussitôt ça y est, ça m'est arrivé, maman le savait, maman sait tout, c'est sûr, je suis morte, je cours derrière eux en hurlant, je cache ma tête dans les jupes de maman, je crie de toutes mes forces : je suis morte... ils ne le savent pas, je suis morte... Mais qu'est-ce que tu as ? Je suis morte, morte, morte, j'ai touché le poteau, voilà, ça y est, la chose horrible, la plus horrible qui soit était dans ce poteau, je l'ai touchée et elle est passée en moi, elle est en moi, je me roule par terre pour qu'elle sorte, je sanglote, je hurle, je suis morte (E, p. 28)

La voix de l'écrivain adulte est complètement absente dans cette scène : c'est la terreur de l'enfant qui parle; sa mort est réelle; les paroles maternelles possèdent une valeur absolue, elles ne sauraient mentir. Le livre entier se déroule autour de cette contradiction fondamentale : la découverte de la violence du langage semble être mise sur le compte de la mère, écrivain elle aussi. Ce n'est pas par hasard que, une fois abandonnée de manière définitive par sa maman, Natacha se retrouvera à réapprendre à écrire, en cherchant à tâtons une langue différente, une langue à elle. D'ailleurs, le rapport avec la mère constitue une ligne directrice ${ }^{48} \mathrm{du}$ récit et explique d'une façon de plus sa caractéristique structurelle principale : la duplicité. L'espace réservé à la figure maternelle - par ses présences et, surtout, par ses maintes absences - est si ample qu'il encourage à voir en cela la source d'inspiration véritable du texte; et certaines déclarations de l'écrivain sur ce point semblent confirmer cette interprétation:

J'ai voulu décrire comment naît la souffrance qui accompagne le sentiment du sacrilège. Quel sacrilège ? Celui qui a consisté pour l'enfant à nier le mensonge originel, à mettre en question la beauté originelle, la suavité, la sublimité, la beauté maternelle qu'elle avait pourtant jusque-là reconnue, absorbée avec volupté. Un sacrilège qui a peut-être déclenché la vie d'un écrivain. ${ }^{49}$

14 Cet acte prométhéen met l'enfant devant l'existence de deux images distinctes de la mère : celle qui existait en dehors de tout raisonnement, faite de tiédeur, légèreté et parfum ; et celle qui naît de sa tendance à juger, à comparer, à mesurer les concepts avec 
les mots. Il suffit de penser à l'épisode de la poupée de coiffeur ${ }^{50}$, à son visage lisse, rose, lumineux, qui obscurcit la perfection de la mère dans l'esprit de la petite fille : « Elle est plus belle que maman» (E, p. 92). À partir de ce moment-là, Natacha vivra dans un sentiment pérenne de culpabilité, elle se convaincra de mériter l'abandon - abandon qui arrivera immanquablement : omnipotence du désir enfantin! Elle ne pourra plus ignorer l'existence d'une deuxième face de la réalité: un revers de toute surface apparente, quelque chose qui cloche et sonne faux dans les mots. Ainsi le double constitue un élément formel, qu'on peut repérer évidemment dans la présence des deux voix - on l'a déjà souligné suffisamment. D'autre part, il est aussi thématisé par une gémination continuelle d'épisodes qui se répètent, en changeant de valeur, dans l'univers de la mère et dans celui du père: c'est là qu'aura lieu le mouvement de libération qui conduit Natacha du premier mot prononcé en autonomie au fixage de sa nouvelle langue sur la page. Comme si on était devant un miroir, on assiste au rejaillissement de plusieurs couples d'oppositions sémantiques: Russie contre France, langue russe contre langue française, intérieur contre extérieur, mère contre père, mère contre belle-mère, familier contre étranger, Natacha (le nom choisi par la mère) contre Tachok (celui préféré par le père), et ainsi de suite, dans une multiplication kaléidoscopique d'espaces linguistiques et géographiques, de maisons partagées et de cœurs brisés ${ }^{51}$. Cette impressionnante symétrie bipartie relève sans doute d'une hantise psychique profonde inscrite dans la personnalité de l'écrivain, une obsession qu'on pourrait analyser d'après la méthode psychocritique proposée par Charles Mauron ${ }^{52}$. Mais elle est surtout liée à l'importance de la réflexion sur le langage chez Sarraute : l'écrivain insiste, on l'a dit, sur la séparation entre le plan de l'émotion et le plan de la traduction de l'idée en paroles. Or c'est là, me parait-il, la clé pour comprendre le charme mystérieux constitué par la duplicité des voix et des images en Enfance. Cette invention originale qui révèle - il faut le répéter une dernière fois - la présence de deux âmes dans le texte, et donne à celui-ci une existence redoublée : sur le versant de la modernité en même temps que sur celui de la tradition.

\section{NOTES}

1. N. Sarraute, Enfance, Paris, Gallimard, «Folio », 1983, p. 7 (dorénavant E). Je tirerai toutes les citations de cette édition; mais il faut, bien sûr, faire référence aussi au volume des CEuvres complètes, éd. publiée sous la direction de J.-Y. Tadié, Paris, Gallimard, «Bibliothèque de la Pléiade », 1996.

2. On sait qu'il n'est pas approprié de parler d'« école » à propos des Nouveaux Romanciers ; mais on peut essayer de ranger des tendances communes, qui sont évidentes, sous le terme de " mouvance » : un terme que R.-M. Allemand a proposé avec autorité dans plusieurs de ses écrits. Cf. notamment R.-M. Allemand et Ch. Milat (dir.), Le "Nouveau Roman » en questions, t. 5, Une "Nouvelle Autobiographie»?, Paris-Caen, Lettres Modernes, La Revue des Lettres Modernes / L'Icosathèque 22, 2004, p. 5.

3. Pour reconstruire l'important réseau tissé entre les écrivains s'inscrivant dans la mouvance du Nouveau Roman, notamment Butor, Robbe-Grillet, Duras et Sarraute, je renvoie aux essais suivants : H. Desoubeaux, « Portrait de l'artiste en jeune singe ou Butor et l'autobiographie : entre 
Sartre et Butor ", dans Mélusine, n. 23, 2003, p.123-129; A. Armel, Marguerite Duras et l'autobiographie, Talence, Le Castor Astral, 1990 ; F. Skutta, «L'Amant de Marguerite Duras : une autobiographie? », dans Cahiers du Cerf XX $X^{e}$, n. 3, 1987, p. 77-90 ; A. Goulet, « La Mémoire dans la "Nouvelle Autobiographie" : Marguerite Duras, Alain Robbe-Grillet ", dans L'École des Lettres II, $1^{\mathrm{er}}$ mars 1995, p. 45-53 ; S. Houppermans, Alain Robbe-Grillet autobiographe, Amsterdam, Rodopi, «Littérature française contemporaine »18, 1993 ; C. Marazza, «Le ambiguità della scrittura nell'autobiografia di Alain Robbe-Grillet », dans Francofonia, n. 15, automne 1988, p. 87-97.

4. Butor a renouvelé plusieurs fois cette expérience par la suite : rappelons Le Retour du Boomerang (1988), Improvisations sur Michel Butor (1993), Curriculum Vitae (1996).

5. À ce propos, Ph. Lejeune soutient : «L'expression "Nouvelle Autobiographie" est en fait plutôt un contre-feu allumé contre l'accusation de "retour à", à laquelle l'air du temps donne tant de vraisemblance ", Ph. Lejeune, " Nouveau Roman et retour à l'autobiographie ", dans M. Contat (éd.), L’Auteur et le manuscrit, Paris, P.U.F., « Perspectives », 1991, p. 51-70, p. 53. L'étiquette de « Nouvelle Autobiographie » proposée par Robbe-Grillet correspondrait en partie à la définition de «autofiction" fournie par S. Doubrovsky. Dans tous les cas (qu'on prenne en considération N. Sarraute, M. Butor, A. Robbe-Grillet, ou M. Duras), «il s'agit de contester l'entreprise autobiographique en plaçant sur le même plan le référentiel et l'imaginaire, ainsi que l'annonce, dès 1977, S. Doubrovsky", comme le disent R.-M. Allemand et Ch. Milat dans " "Nouveau Roman". "Nouvelle Autobiographie" ?», dans R.-M. Allemand et Ch. Milat (dir.), Le "Nouveau Roman » en questions, t. 5, Une « Nouvelle Autobiographie » ?, op. cit., p. 14.

6. Pour un discours plus détaillé sur la fortune du modèle rousseauien, qui a fixé les règles du genre autobiographique et la " grammaire » du récit d'enfance, je renvoie à S. Zatti, Raccontare la propria infanzia, postface à F. Orlando, Infanzia, Memoria e Storia da Rousseau ai Romantici, Pisa, Pacini Editore, 2007, p. 310.

7. Mais je rappelle que B. Vercier s'est déjà engagé sur cette voie. Voici l'une de ses considérations à titre d'exemple : « Les clichés ont la vie dure : certains critiques se sont étonnés, au moment de la publication d'Enfance, de ce que Nathalie Sarraute, romancière du Nouveau Roman, ait pu écrire une autobiographie, comme si le Nouveau Roman avait été non pas la recherche d'un nouveau réalisme par un groupe d'écrivains bien différents les uns des autres, mais une école rigide de romanciers se contentant de décrire "objectivement" et au présent le monde des choses extérieures; comme si, surtout, l'Autobiographie n'était pas, chaque fois qu'elle est réussie, le fruit d'une œuvre, c'est-à-dire d'une écriture élaborée tout au long de cette œuvre » (B. Vercier, « (Nouveau) Roman et Autobiographie : Enfance de Nathalie Sarraute», dans French Literature Series, n. XII, 1985, p. 162-170, p. 162).

8. Je pense évidemment à son Ou bien... ou bien (1843), connu aussi sous le nom révélateur de L'alternative.

9. Pour le dire en d'autres mots: «La position distante, celle qui ordonne, juge et nomme, la saisie encyclopédique du monde - toutes ces sentences verbales par exemple qui tombent dans le cosmos préverbal de l'enfant sont représentées comme inadéquates à son expérience » (D. Keller, " Autobiographie et authenticité chez Nathalie Sarraute », dans Le "Nouveau Roman » en questions, t. 5, op. cit., p. 57-74, p. 68).

10. À ce propos, K. Wei parle d'une "mise en scène des repentirs", en utilisant le terme «repentirs» dans le sens qu'il a dans la terminologie de la peinture, où il désigne des changements apportés en cours d'exécution, non pas après coup, mais en cours, au fur et à mesure que le dessin prend forme. De la même manière, N. Sarraute met en scène le processus de l'écriture au fur et à mesure qu'il est en train de se faire (et ses tropismes - pourrait-on ajouter mettent en scène les pensées dans l'acte même de se composer). Cf. K. Wei, «Pluralité des voix et repentirs autobiographiques : une lecture d'Enfance de Nathalie Sarraute », dans Études françaises (Pascal Quignard, ou le noyau incommunicable), vol. 40, n. 2, Les Presses de l'Université de Montréal, 2004, p. 101-114. 
11. J.-J. Rousseau, Essai sur l'origine des langues dans Écrits sur la musique, la langue et le théâtre. Textes historiques et scientifiques, Paris, Gallimard, « Bibliothèque de la Pléiade », 1995, p. 380.

12. M. Gosselin, Enfance de Nathalie Sarraute, Paris, Gallimard, « Foliothèque », 1996, p. 14.

13. D. Rabaté, «Le Présent de la parole», dans Critique, Nathalie Sarraute ou l'usage de l'écriture, janvier-février 2002, p. 51-60, p. 56.

14. M. Gosselin-Noat, "Nathalie Sarraute et la recherche de la vérité », dans Critique, Nathalie Sarraute ou l'usage de l'écriture, cit., p. 22-35, p. 24.

15. N. Sarraute, L'Ère du soupçon, Paris, Gallimard, 1956, p. II.

16. En fait, une autobiographie qui critique l'autobiographie en passant par « les cicatrices de la langue », comme le remarque M. Calle-Gruber dans « Le fil de soie » : M.-L. Mallet (dir.), L'animal autobiographique. Autour de Jacques Derrida, Paris, Galilée, 1998, p. 61-84, p. 77. Y. Immoune parle, de son côté, de "renouveau du récit autobiographique", un renouveau qui passe par le déplacement $d u$ focus d'un paradigme à un autre: du niveau référentiel typique de l'autobiographie classique au niveau langagier. Cf. Y. Immoune, «Éléments du renouveau du récit autobiographique dans Enfance de Nathalie Sarraute: du paradigme référentiel au paradigme langagier ", dans A. Bererhi (dir.), L'autobiographie en situation d'interculturalité, Actes du colloque international des 9-10-11 décembre 2003, t. II, Alger, Université d'Alger, 2004, p. 437-451.

17. La définition du concept d'authenticité ne va évidemment pas sans problèmes. On ne saurait certainement expliquer en quelques lignes tous les enjeux de cette catégorie axiologique. Mais, pour en avoir un aperçu, il faudra réfléchir au moins sur l'existence d'une notion qui est à la fois proche et nettement distincte de celle-là : la véridicité. En signant le pacte autobiographique, le mémorialiste s'engage à garantir le caractère authentique de sa narration : il n'écrira que ce qui est vrai pour lui, vrai pour le sujet, vrai pour la conscience qui s'analyse. La véridicité reste plutôt la visée de l'historien - l'étude des actions humaines s'opposant en cela à celle des sentiments, des mouvements du cœur, des élans de l'âme. Encore faut-il ajouter que l'idée d'authenticité est dynamique et répond aux sollicitations du moment historique: si Sarraute en recherche une actualisation ainsi que Rousseau, elle ne le fait pas pour autant par les mêmes moyens, elle n'obtient pas les mêmes résultats, elle ne construit pas les mêmes effets de transposition.

18. D. Keller, « Autobiographie et authenticité chez Nathalie Sarraute », op. cit., p. 69.

19. F. Orlando, Infanzia, Memoria e Storia da Rousseau ai Romantici, cit. Malheureusement, le livre n'a jamais été traduit en français. Il s'agit d'une sorte d'histoire de la littérature autobiographique française et, plus précisément, de la parabole accomplie par le souvenir d'enfance entre Rousseau et $G$. Sand. Orlando a indiqué toute une série de constantes qui qualifient le thème; j'en résume ici les principales (qu'il introduit à la p. 11), cinq oppositions binaires dont je me servirai plusieurs fois au cours de mon analyse: 1) souvenirs d'enfance gratuits vs souvenirs motivés par la narration ; 2) objets du souvenir vs processus du souvenir ; 3 ) « regard en arrière » $v s ~ «$ regard d'en bas »;4) souvenirs retrouvés les yeux fermés vs présence de traces matérielles du souvenir ; 5) abandon au souvenir vs critique du souvenir.

20. Lorsqu'on parle de souvenirs d'enfance gratuits (ce qu'on fera à maintes reprises dans cette analyse), il faudrait toujours préciser le caractère problématique - apparent ou relatif - de toute gratuité. Mais, dans une perspective historiciste, on peut bien récupérer cette étiquette. À condition seulement que l'on pose l'accent sur l'économie interne au texte, et/ou sur la perception de l'auteur et de ses lecteurs durant une période historiquement déterminée. À la fin $\mathrm{du} \mathrm{XVIII}^{\mathrm{e}}$ siècle, il n'était licite de se raconter que sous prétexte d'enseigner une vérité d'ordre moral : en transgressant le tabou, Rousseau « invente » l'autobiographie moderne. Dans ce senslà, on pourrait voir le premier des binômes orlandiens (souvenirs d'enfance gratuits vs souvenirs motivés par la narration) comme celui qui les contient tous.

21. F. Orlando, Les objets désuets dans l'imagination littéraire: ruines, reliques, raretés, rebuts, lieux inhabités et trésors cachés, Paris, Garnier, « Classiques », 2010, p. 460.

22. R. Girard, Mensonge romantique et vérité romanesque, Paris, Grasset, 1961, p. 70. 
23. S. Freud, Un souvenir d'enfance de Léonard de Vinci, tr. fr. M. Bonaparte, Paris, Gallimard, «Idées », 1936, p. 63.

24. C. Ginzburg, "Traces. Racines d'un paradigme indiciaire ", dans Mythes, Emblèmes, Traces. Morphologie et Histoire, tr. fr. M. Aymard, Paris, Flammarion, « Nouvelle Bibliothèque Scientifique », 1989, p. 139-180.

25. C'est Auerbach qui a étudié tout cela admirablement. Je pense évidemment à Mimésis, la représentation de la réalité dans la littérature occidentale, tr. fr. C. Heim, Paris, Gallimard, 1968. Voir particulièrement les p. 450-488 (chapitre « À l'Hôtel de la Mole »).

26. Cf. F.-R. de Chateaubriand, Mémoires d'outre-tombe, t. I, Paris, Garnier, "Classiques ", 1989-1998, p. 128.

27. M. Gosselin, Enfance de Nathalie Sarraute, cit., p. 21.

28. V. Minogue a choisi pour son article sur Enfance un titre qui pourrait bien en être la description: "Fragments of a Childhood: Nathalie Sarraute's Enfance ", dans Romance Studies, n. 9, 1986, p. 71-83.

29. Le terme est utilisé par K. Wei dans «Pluralité des voix et repentirs autobiographiques : une lecture d'Enfance de Nathalie Sarraute », cit., p. 101.

30. C. Burgelin, Les parties de dominos chez Monsieur Lefèvre. Perec avec Freud-Perec contre Freud, Saulxures, Circé, 1996, p. 213.

31. Ph. Lejeune, Le pacte autobiographique, Paris, Seuil, 1975, p. 14.

32. Ch. Doumet, "Le stéréoscope de Nathalie Sarraute", dans Nathalie Sarraute ou l'usage de l'écriture, Critique, Janvier-Février 2002, p.81-93; E. D. Valenta, Doubled selves and fractured childhoods : a study of the "récit d'enfance » in Nathalie Sarraute, Agota Kristof and Claude Esteban, Ann Arbor, Mi, UMI, Bell \& Howell Company, 1991.

33. F. Orlando, Infanzia, Memoria e Storia da Rousseau ai Romantici, cit., p. 10-11.

34. Ibid, p. 10. Je traduis de l'édition italienne.

35. D. Keller l'a très bien expliqué : «Les expériences toujours nouvelles, toujours différentes, viennent s'infiltrer à l'intérieur du Je sans qu'un mot ne les intercepte aux limites de ce Je », " Autobiographie et authenticité chez Nathalie Sarraute », cit., p. 68.

36. Sarraute préfère ce terme à celui d'inconscient.

37. V. Minogue, «Danger de mort: la mort et ses avatars dans l'œuvre de Nathalie Sarraute », dans Critique, Nathalie Sarraute ou l'usage de l'écriture, cit., p. 119-132, p. 120.

38. On peut citer entre autres L'autobiographie en France, Paris, Armand Colin, 1998; Le pacte autobiographique, Paris, Seuil, 1975 ; Signes de vie. Le pacte autobiographique 2, Paris, Seuil, 2005.

39. Ph. Lejeune, "Paroles d'enfance", dans Revue des Sciences Humaines, t. LXXXXIII, n. 217, janvier-mars 1990, p. 23-40, p. 23.

40. Ibid., p. 38.

41. N. Sarraute, Entre la vie et la mort, Paris, Gallimard, « Folio », 1968, p. 10.

42. «[...] il était temps de songer au départ et auparavant de se donner le plaisir de regarder un instant en arrière.", Stendhal, Euvres intimes, édition établie par V. Del Litto, t. II, Paris, Gallimard, «Bibliothèque de la Pléiade », 1982, p. 628. «J'ai vu tout cela d'en bas comme un enfant [...]», ibid., p. 638. C'est moi qui souligne.

43. Ce sont les termes de la deuxième distinction opérée par Orlando : cf. Infanzia, Memoria e Storia da Rousseau ai Romantici, cit., p. 9-10.

44. Avant qu'ils disparaissent... était aussi le titre que l'écrivain avait choisi, au début, pour son autobiographie.

45. Cf. A. Clayton, "Coucou... attrapez-moi... », dans Revue des Sciences Humaines, t. LXXXXIII, n. 217, janvier-mars 1990, p. 9-22, p. 9.

46. Elle observe encore : «Finalement, le motif de l'enfance, lié à la sensibilité tropismique et artistique, lié à une attitude devant le monde qu'on peut qualifier d'authentique, tient ensemble toutes ces lectures [elle se réfère à ses propres conclusions, mais nous aussi, nous pourrions le 
dire] qu'on vient d'esquisser.", D. Keller, "Autobiographie et authenticité chez Nathalie Sarraute ", cit., p. 72.

47. Cf. E, p. 211.

48. Ce qu'affirme aussi N. de Courson dans Nathalie Sarraute. La Peau de maman, Paris, L'Harmattan, 2010.

49. V. Forrester, «Portrait de Nathalie », dans Le Magazine littéraire, n. 196, juin 1983, p. 18-21, p. 19.

50. E, p. 91-103.

51. Pour une confrontation plus ponctuelle, je renvoie à E. D. Valenta, Doubled selves and fractured childhoods, cit., p. 115 et sq.

52. Ch. Mauron, Des métaphores obsédantes au mythe personnel. Introduction à la psychocritique, Paris, José Corti, 1963.

INDEX

Mots-clés : Sarraute (Nathalie), Enfance, autobiographie, Rousseau (Jean-Jacques), souvenir d'enfance, voix stéréophoniques, binômes antinomiques, Orlando (Francesco) 